\title{
A practical criterion of irreducibility of multi-loop Feynman integrals.
}

\author{
P. A. BAIKOV ${ }^{1}$ \\ Skobeltsyn Institute of Nuclear Physics, Moscow State University, \\ Moscow 119992, Russia
}

\begin{abstract}
A practical criterion for the irreducibility (with respect to integration by part identities) of a particular Feynman integral to a given set of integrals is presented. The irreducibility is shown to be related to the existence of stable (with zero gradient) points of a specially constructed polynomial.
\end{abstract}

The growing accuracy of high energy physics experiments demands the calculation of higher order quantum corrections. The latter, in turn, are expressed through multi-loop Feynman integrals. Most of the methods for their calculations are designed for the integrals with a monomial of momenta in the numerator. On the other hand, in practice one can face thousands and even millions of such integrals in one particular physical problem. Fortunately, it is possible to reduce this number to around a few dozens by using integration by part identities [1]. These identities can be solved in some cases by constructing recursive algorithms $[1,2]$ or by "brute computer force" with the Laporta algorithm [3]. In any case, it is useful to know if the given integral can be related to simpler integrals (with some denominators missing). A corresponding criterion of irreducibility was proposed in [4]: the irreducibility was related to the existence of a special solution of the recurrence relations; a recipe to construct such solutions was suggested. Unfortunately, the recipe in the form of [4] required some "hand work" which becomes very undesirable for the reallife problems (too many cases to be considered). So in this paper we propose a more practical version of this criterion which amounts to the study of zerogradient points of some polynomial and hence can be easily implemented with a computer. We start with examples, then formulate the criterion and finally present the list of the irreducible four-loop massless propagator integrals found with the help of our criterion.

1 Supported in part by INTAS (grant 03-51-4007) and RFBR (grant 05-02-17645); e-mail: baikov@theory.sinp.msu.ru

Preprint submitted to Elsevier Science 21 November 2018 


\section{Examples.}

Consider two massless 4-loop propagator integrals:
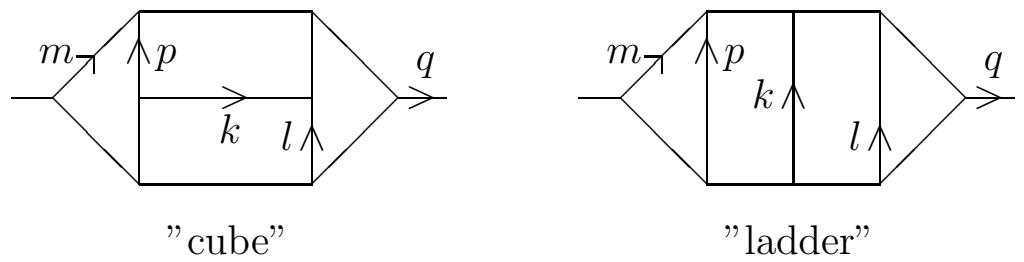

The "ladder" integral consist of triangles and thus, according to "triangle rule" [1], can be related to the combination of simpler integrals; the reducibility of the "cube" diagram is unclear. To study this question we are going to associate with every multi-loop integral some polynomial, whose properties reflect its irreducibility.

Let us calculate the determinant $P=\operatorname{det}\left(p_{i} p_{k}\right)\left(p_{i}=q, m, p, k, l\right)$ under the condition that all lines are "on mass-shell", that is corresponding squared momentum are equal to zero. In total there are 15 scalar products ("external" $q^{2}$ and 14 "internal" involving loop momenta) with 11 constraints, so we can express 11 "internal" scalar products through others. For "cube" it is convenient to choose $p q, l p, l q, q^{2}$ as independent:

$P_{c u b}=-\left(2 l p \cdot l q+2 l p \cdot p q+l p \cdot q^{2}-2 l q \cdot p q\right) \cdot l p \cdot l q \cdot p q$.

For "ladder" let us choose $k l, k p, l p, q^{2}$ as independent:

$P_{l a d}=\left(k l \cdot k p-l p \cdot q^{2} / 2\right) \cdot k l \cdot k p$.

For each polynomial let us check if there are points where all derivatives with respect to the "internal" scalar products are zero, but the polynomial itself and the determinant of second derivatives are not zero (in short "non-zero stable points"). The "ladder" polynomial has no such points, and the "cube" has one point: $l p=-9 q^{2} / 20, p q=-3 q^{2} / 10, l q=-3 q^{2} / 10$. As we will see later, this means irreducibility of the "cube" integral.

\section{Criterion in general form.}

Now let us explain why non-zero stable points of some polynomial are related to the irreducibility. Assume that there is a set of equations which relate the function $B(\underline{n})$ (keeping in mind Feynman integrals as functions of degrees of propagators and irreducible numerators) with arguments shifted by \pm 1 :

$$
R\left(I^{-}, I^{+}\right) B(\underline{n})=0
$$


where $(\underline{n})$ (and others underlined arguments) is $\left(n_{1}, . ., n_{N}\right), \mathbf{I}_{a}^{-} B\left(. ., n_{a}, ..\right) \equiv$ $B\left(. ., n_{a}-1, ..\right)$ and $\mathbf{I}_{a}^{+} B\left(. ., n_{a}, ..\right) \equiv n_{a} B\left(. ., n_{a}+1, ..\right)$. Since $\mathbf{I}^{-}$and $\mathbf{I}^{+}$do not commute $\left(\left[\mathbf{I}_{a}^{-}, \mathbf{I}_{b}^{+}\right]=\delta_{a b}\right)$, for definiteness we place all $\mathbf{I}^{+}$to the right of $\mathbf{I}^{-}$.

We want to check the possibility to represent, using the relations (1), a given $B\left(\underline{n}^{(0)}\right)$ as linear combination of $\left\{B\left(\underline{n}^{(i)}\right), i \in(1, \ldots, k)\right\}$ :

$$
0 \stackrel{?}{=} B\left(\underline{n}^{(0)}\right)-\sum_{i=1}^{k} f_{i} B\left(\underline{n}^{(i)}\right)
$$

The relation (2), if it exists, should be a combination of the relations (1) for various values of $\underline{n}$, so any solution of (1) should also obey (2). Suppose we are able to construct the special solution of (1) $s(\underline{n})$ with the properties

$$
s\left(\underline{n}^{(0)}\right) \neq 0, \quad s\left(\underline{n}^{(i)}\right)=0 \quad \text { for } i \in(1, \ldots, k) .
$$

This $s(\underline{n})$ evidently cannot obey (2) and hence (2) cannot be the combination of (1). So we got a sufficient criterion of irreducibility in general form [4]: the recurrence relations (1) cannot relate the $B\left(\underline{n}^{(0)}\right)$ to linear combination of the set $\left\{B\left(\underline{n}^{(i)}\right), i \in(1, \ldots, k)\right\}$ if a partial solution of (1) with properties (3) exits.

\section{Formal solutions of the recurrence relations.}

Let us construct the solutions of the relations (1) as formal series in $1 / D$ :

$$
B(\underline{n}, D)=\frac{1}{D^{k_{0}(\underline{n})}} \sum_{k=0}^{\infty} \frac{B^{(k)}(\underline{n})}{D^{k}} .
$$

To be precise, we will present an algorithm for calculation of the $B^{(k)}$ such that for any $\underline{n}$ and arbitrary large $N$, sufficiently long partial sum of (4) will obey (1) up to terms $1 / D^{N}$.

As an example, let us consider the recurrence relations

$$
0=(k-2 D+2) B_{k+1}+2 a(k-D+1) B_{k}+k b B_{k-1}
$$

generated by the integral

$$
B_{k}=\int_{-\infty}^{\infty} x^{k}\left(x^{2}+2 a x+b\right)^{-D} d x, \quad k \geq 0, k+1-2 D<0, b>a^{2}
$$


After the substitution $x=\tilde{x} / \sqrt{D}-a$ integral (6) can be represented as

$$
\begin{aligned}
& B_{k}=\sum_{l=0}^{k} \frac{\Gamma(k+1)}{\Gamma(l+1) \Gamma(k-l+1)}(-a)^{k-l} D^{-l / 2-1 / 2} \tilde{B}_{l} \\
& \tilde{B}_{k}=\int_{-\infty}^{\infty} \tilde{x}^{k}\left(\frac{\tilde{x}^{2}}{D}+b-a^{2}\right)^{-D} d \tilde{x}
\end{aligned}
$$

The $\tilde{B}_{k}$ obeys the recurrence relations

$$
0=\left(k\left(b-a^{2}\right) \tilde{B}_{k-1}-2 \tilde{B}_{k+1}\right)+\frac{1}{D}(k+2) \tilde{B}_{k+1}=R_{k}^{0}(\tilde{B})+\frac{1}{D} R_{k}^{1}(\tilde{B}) .
$$

Inserting $\tilde{B}_{k}=\sum_{l=0}^{\infty} \tilde{B}_{k}^{(l)} / D^{l}$ we obtain the following equations for $\tilde{B}_{k}^{(l)}$ :

$$
0=R_{k}^{0}\left(\tilde{B}^{(0)}\right), \quad 0=R_{k}^{0}\left(\tilde{B}^{(l+1)}\right)+R_{k}^{1}\left(\tilde{B}^{(l)}\right) \quad l>0 .
$$

If $b-a^{2} \neq 0$, these equations have nontrivial solution and we obtain the desired solution of the (5) as formal series in $1 / D$. Note that we can forget about integral (6) and study the relation (5) with the help of substitution (7). In particular if $b \leq a^{2}$ the integrand of (6) has poles on the real axes, but the substitution (7) does not depend on the definition (and even on the convergence) of the integral. From other side, the "integral representation" language is more convenient, so in general case we will construct solutions (3) presenting necessary manipulations in this way, keeping in mind that for formal proofs we can translate all steps to "recurrence relations" language.

\section{Formal solutions in general case.}

To construct the integral representation for $s(\underline{n})(3)$ let us try $[5]$ :

$$
s(\underline{n})=\int \frac{d x_{1} \ldots d x_{N}}{x_{1}^{n_{1}} \ldots x_{N}^{n_{N}}} g(\underline{x}) .
$$

The action of $R\left(I^{-}, I^{+}\right)$on (9) leads to

$$
\begin{aligned}
R\left(I^{-}, I^{+}\right) s(\underline{n}) & =\int d x_{1} \ldots d x_{N} g(\underline{x}) R\left(\underline{x},-\partial_{\underline{x}}\right) \frac{1}{x_{1}^{n_{1}} \ldots x_{N}^{n_{N}}} \\
& =\int \frac{d x_{1} \ldots d x_{N}}{x_{1}^{n_{1}} \ldots x_{N}^{n_{N}}} R^{\prime}\left(\underline{x}, \partial_{\underline{x}}\right) g(\underline{x})+(\text { surface terms }),
\end{aligned}
$$


where we use integration by part in second equation and denote by $R^{\prime}$ the combination of $\underline{x}$ and $\partial_{\underline{x}}$ acting on $g(\underline{x})$. Now, if one chooses $g(\underline{x})$ so that

$$
R^{\prime}\left(\underline{x}, \partial_{\underline{x}}\right) g(\underline{x})=0
$$

and chooses the integration contours such that the surface terms vanish, one arrives to the function $s(\underline{n})$ such that $R\left(I^{+}, I^{-}\right) s(\underline{n})=0$.

As shown in [5], the (11) can be solved for the general case of a multi-loop Feynman integral with arbitrary number of legs and with arbitrary masses, and the corresponding $g(\underline{x})$ can be represented as a product of two polynomials in $\underline{x}$, each polynomial raised to non-integer degree. Then, the recurrence relations for $L$-loop $R$-leg integrals is a partial case of the recurrence relations for $R+L-1$ tadpole integrals [6], thus we limit ourselves by the tadpole case.

Consider an $L$-loop tadpole Feynman integrals in dimensional regularization:

$$
B(\underline{n}) \equiv \int \frac{d^{D} p_{1} \ldots d^{D} p_{L}}{D_{1}^{n_{1}} \ldots D_{N}^{n_{N}}}
$$

where $p_{i}(i=1, \ldots, L)$ are loop momenta and

$$
D_{a}=\sum_{i=1}^{L} \sum_{j=1}^{L} A_{a}^{i j} p_{i} \cdot p_{j}-m_{a}^{2} \quad(a=1, \ldots, N=L(L+1) / 2)
$$

are propagators $\left(n_{a}>0\right)$ or irreducible numerators $\left(n_{a} \leq 0\right)$. Consider some integral $B\left(\underline{n}^{(0)}\right)$ with the set of positive indexes ("lines") $S: a \in S$ if $n_{a}^{(0)}>0$. Normally, the integrals with less number of lines are easier to calculate, so let us check if it is possible to relate this integral to integrals with some lines missing. For that we should try to construct $s(\underline{n})$ such that $s(\underline{n})=0$ if at least one of the $n_{a \in S} \leq 0$. Eq. (9) in this case will read [5]

$$
s(\underline{n})=\int \frac{d x_{1} \ldots d x_{N}}{x_{1}^{n_{1}} \ldots x_{N}^{n_{N}}} P(\underline{x})^{(D-L-1) / 2},
$$

where $P(\underline{x})=\operatorname{det}_{(k l)}\left(\sum_{a=1}^{N} \tilde{A}_{a}^{k l}\left(x_{a}+m_{a}^{2}\right)\right)$ is polynomial in $\underline{x}$ and $\tilde{A}_{a}^{k l}$ is the matrix inverse of $A_{a}^{k l}$, i.e. $\sum_{a=1}^{N} \tilde{A}_{a}^{i j} A_{a}^{k l}=\left(\delta_{i k} \delta_{j l}+\delta_{i l} \delta_{j k}\right) / 2$. In other words, to construct $P$ one should calculate the determinant of scalar products of the loop momenta and then substitute the scalar products by the combinations of propagators and numerators obtained by inverting (12).

Let us choose the integration contours for $x_{a \in S}$ as small circles around zero. In this case, according to Cauchy's theorem, the integrations will lead to the 
$\left(n_{a}-1\right)^{t h}$ coefficient in the Taylor expansion of the integrand:

$$
\left.s(\underline{n}) \propto \int \prod_{a \notin S}\left[\frac{d x_{a}}{x_{a}^{n_{a}}}\right] \prod_{a \in S}\left[\frac{\partial_{a}^{n_{a}-1}}{\left(n_{a}-1\right) !}\right] P(\underline{x})^{(D-L-1) / 2}\right|_{x_{a \in S}=0} .
$$

In the case when the number of lines is equal to the number of integrations in (13) the (14) has no integrations and (if $\left.P(\underline{x})\right|_{x_{a \in S}=0} \neq 0$ ) the construction of the $\mathrm{s}(\underline{\mathrm{n}})$ is completed; in the general case, we need a way to define the integration over the remaining $x_{a \notin S}$. As the result of the Taylor expansion, the original $s(\underline{n})$ will be linear combination of the terms of the following type:

$$
\begin{aligned}
s(\underline{n}) & =\sum_{k_{a \notin S}} C_{k_{a \notin S}} \int \prod_{a \notin S}\left[d x_{a} x_{a}^{-k_{a}}\right] \tilde{P}(\underline{x})^{D^{\prime} / 2}, \\
\tilde{P} & \underline{x})=\left.P(\underline{x})\right|_{x_{a \in S}=0}, \quad D^{\prime}=D-L-1-\sum_{a \in S}\left(n_{a}-1\right) .
\end{aligned}
$$

Let us expand (15) in the formal series in the limit $D^{\prime} \rightarrow-\infty$. Assume there exists a point $\underline{x}^{0}$ with:

$$
\tilde{P}\left(\underline{x}^{0}\right) \neq 0, \quad \partial_{a \notin S} \tilde{P}\left(\underline{x}^{0}\right)=0, \quad \operatorname{det}_{a b}\left(\partial_{a \notin S} \partial_{b \notin S} \tilde{P}\left(\underline{x}^{0}\right)\right) \neq 0 .
$$

After the substitution $\underline{x}=\underline{x}^{0}+\underline{x}^{\prime} / \sqrt{-D^{\prime}}$

$$
\begin{aligned}
\tilde{P}(\underline{x})^{\frac{D^{\prime}}{2}} & \approx\left(\tilde{P}\left(\underline{x}^{0}\right)-\sum_{a, b \notin S} \frac{x_{a}^{\prime} x_{b}^{\prime}}{2 D^{\prime}} \partial_{a} \partial_{b} \tilde{P}\left(\underline{x}^{0}\right)+O\left(\left(-D^{\prime}\right)^{-3 / 2}\right)\right)^{\frac{D^{\prime}}{2}} \\
& \approx \tilde{P}\left(\underline{x}^{0}\right)^{\frac{D^{\prime}}{2}}\left(e^{-\frac{1}{4 \tilde{P}\left(\underline{x}^{0}\right)} \sum_{a, b \notin S} x_{a}^{\prime} x_{b}^{\prime} \partial_{a} \partial_{b} \tilde{P}\left(\underline{x}^{0}\right)}+O\left(\left(-D^{\prime}\right)^{-1 / 2}\right)\right) .
\end{aligned}
$$

The Gaussian integrations can be performed by the formula

$$
\int \prod_{a=1}^{M}\left(d x_{a} x_{a}^{k_{a}}\right) e^{\left(-x_{a} C^{a b} x_{b}\right)}=\left.\frac{\pi^{M / 2}}{\operatorname{det}(C)^{1 / 2}} \prod_{a=1}^{M}\left(\frac{d}{d t_{a}}\right)^{k_{a}} e^{\left(\frac{1}{4} t_{a} C^{-1, a b} t_{b}\right)}\right|_{t_{a}=0} .
$$

Finally, expansion in $D^{\prime} \rightarrow-\infty$ can be easily recalculated to $D \rightarrow-\infty$.

Eq. (16) defines the practical version of the criterion: if (16) is valid, then the eqs. $(14,15,17,18)$ define the algorithm for calculating $s(\underline{n})$ with properties $(3)$ thus proving the irreducibility of the integral $B\left(\underline{n}^{(0)}\right)$. Note that $P$ in (13) is $\operatorname{det}\left(p_{i} p_{k}\right)$ where scalar products are expressed through linear combinations of $\underline{x}$, and hence $\tilde{P}$ in (15) is equivalent to $\operatorname{det}\left(p_{i} p_{k}\right)$ with lines "on-shell", as it was considered in the examples. 
The equation (16) and hence (15) can have several solutions $s^{(p)}(\underline{n})$. This corresponds to possibility to find several integrals with the same set of lines (differ by number of dots or by numerators) which are irreducible as to each other, as to simpler integrals. To fix these irreducible integrals, let us choose some set of points in " $\underline{n}$ "-space $\underline{n}^{(q)}$ such that the matrix $M_{q}^{p}=s^{(p)}\left(\underline{n}^{(q)}\right)$ has inverse matrix $\bar{M}_{p}^{q}$. Then solutions $\bar{M}_{p}^{q} s^{(p)}$ will be "diagonal" in the sense (3) and hence the set $\underline{n}^{(q)}$ will define irreducible integrals.

The application of the criterion (16) to the four-loop massless propagator integrals (or equivalently five-loop tadpole with one massive line) results in the following list of integrals which cannot be reduced to integrals with a smaller number of lines (for each of the topologies depicted here only a single irreducible integral exists):
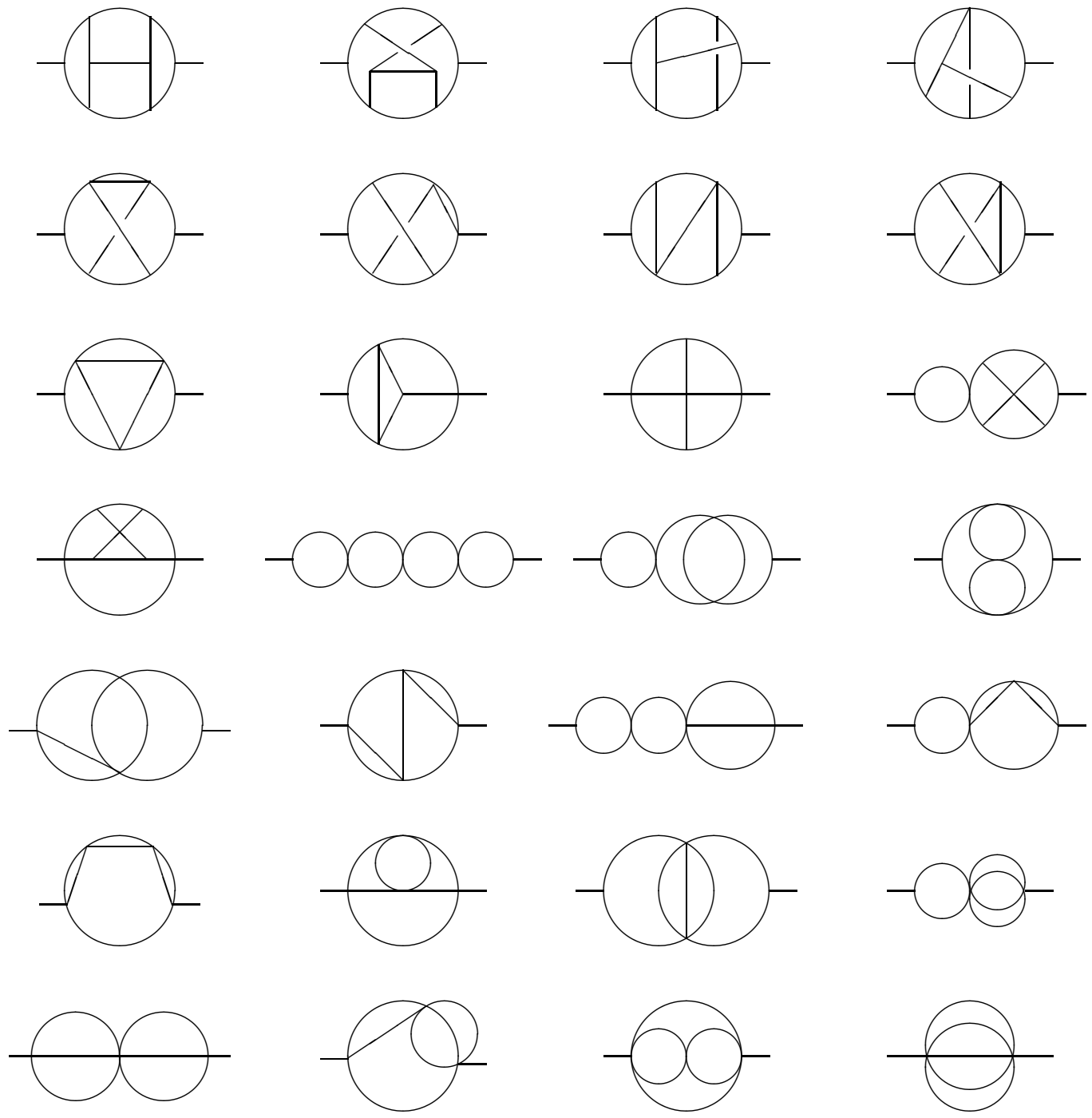


\section{Final remarks.}

The criterion (16) is easy to implement in the case when the polynomial $\tilde{P}$ in (15) depends only on a few variables (large number of lines in the integral). In the most easy case when number of lines is equal to number of "internal" scalar products the irreducibility will follow from $P(0) \neq 0$. In the case of a small number of lines (many variables in $\tilde{P}$ ) it may be difficult to solve (16) explicitly. In this case it is instructive to check (using Groebner basis) if the polynomial $\tilde{P}$ is reducible to polynomials $\partial_{a \notin S} \tilde{P}$. If so, then $\partial_{a \notin S} \tilde{P}\left(\underline{x}^{0}\right)=0$ leads to $\tilde{P}\left(\underline{x}^{0}\right)=0$ and there no non-zero stable points. On the other hand, reducibility of the integrals with a small number of lines in many cases can be checked directly (with the triangle rule or by checking subdiagrams).

And finally, let us address the question what happens if (16) is not satisfied. Formally this case is unclear, but in practice (at least for massless propagator integrals up to 4-loop and for zero-scale 2-loop propagator integrals [7]) it corresponds to reducible integrals.

The author are grateful to K.G.Chetyrkin, R.Harlander, J.H.Kuehn, Y.Schroder and O.V.Tarasov for the careful reading of the manuscripts and useful discussions.

\section{References}

[1] K.G. Chetyrkin and F.V. Tkachov, Nucl. Phys. B192 (1981) 159;

F.V. Tkachov, Phys. Lett. B100 (1981) 65.

[2] D.J. Broadhurst, Z. Phys. C54 (1992) 599;

L.V. Avdeev, Comput. Phys. Commun. 98 (1996) 15;

O.V. Tarasov, Nucl. Phys. B502 (1997) 455;

O.V. Tarasov, Acta Phys. Polon. B29 (1998) 2655;

V.A.Smirnov and O.L. Veretin, Nucl. Phys. B566 (2000) 469;

C. Anastasiou, T. Gehrmann, C. Oleari, E. Remiddi and J.B. Tausk, Nucl. Phys. B580 (2000) 577;

A.V.Smirnov and V.A.Smirnov, hep-lat/0509187.

[3] S. Laporta and E. Remiddi, Phys. Lett. B379 (1996) 283;

P. Mastrolia and E. Remiddi, Nucl. Phys. Proc. Suppl. 89 (2000) 76.

[4] P.A. Baikov, Phys. Lett. B474 (2000) 385; hep-ph/9912421.

[5] P.A. Baikov, Phys. Lett. B385 (1996) 404; Nucl. Inst. \& Meth. A389 (1997) 347.

[6] P.A. Baikov and V.A. Smirnov, Phys. Lett. B477 (2000) 367; hep-ph/0001192.

[7] J. Fleischer, M.Yu. Kalmykov and A.V. Kotikov, Phys. Lett. B462 (1999) 169. 